\title{
El poder del ejemplo: un análisis reticular del rodaje de una escena cinematográfica
}

\author{
Dafne Muntanyola - Carlos Lozares - Departament de Sociología (UAB) ${ }^{1}$
}

\begin{abstract}
Resumen
Este artículo se propone explicar el rodaje de una escena cinematográfica, definido como proceso de representación, integrado por agentes intencionales y recursos representacionales. En concreto, el análisis reticular da una representación externa de una red técnico-funcional, y explicita sus dimensiones de coordinación, sincronización y complejidad, en tanto que proceso productivo. Con relación a la perspectiva sociológica, en la que haremos una síntesis entre el marco conceptual propuesto y el material empírico recogido en el entorno, nos centraremos sobre todo en dos mecanismos de dominación. Estos configuran el rodaje como un intercambio de recursos dominados y dominantes, partiendo de una estructura de distribución desigual entre los agentes. En definitiva, se explora la utilidad de en análisis reticular para entender el desarrollo objetivo del rodaje cinematográfico , a la vez que se reivindica la necesidad de analizar las relaciones de poder presentes en todo proceso social.
\end{abstract}

Palabras clave: Proceso de representación - intencionalidad - recurso representacional - red técnico-funcional.

\begin{abstract}
The present article explains the shooting of a single movie scene, defined as a process of representation, with intentional agents and representational resources. Network Analysis as presented gives us an external representation of a Technical and Functional network. Furthermore, three dimensions of the observed productive process are put forward: coordination, synchronization, and complexity. Two mechanisms of domination are extracted from the suggested synthesis between our theoretical framework and the empirical material gathered. Such mechanisms shape the filmmaking process as an exchange of dominated and dominant resources, given an unequal distribution structure. In all, Network Analysis is seen from a sociological perspective as useful, in order to understand the objective development of the filmmaking process. Nevertheless, it is claimed that power relations need to be analyzed further, as central elements in any social process.
\end{abstract}

Key Words: Representational Process - Intentionality - Representational Resource - Technical and Functional Network.

\footnotetext{
${ }^{1}$ Enviar correspondencia a dafne.muntanyola@uab.es; carlos.lozares@uab.es
} 


\section{I ntroducción}

Este artículo tiene como objetivo explicar una situación de producción de conocimiento interactivo, como es el rodaje de una escena cinematográfica, a partir del análisis de redes sociales. Más allá de los resultados obtenidos, se quiere mostrar cómo la misma naturaleza instrumental y heterogénea de las redes sociales necesita una previa construcción teórica del objeto de estudio, esto es, una previa explicación teórica del funcionamiento de un rodaje cinematográfico.

Por lo tanto, en un primer apartado, se justificará la perspectiva micro adoptada a partir de la observación de un rodaje cinematográfico de forma casi-etnográfica y se dará una definición del proceso de representación cinematográfico como una actividad social intencional y representacional. Una vez definidos los conceptos de representación y de intencionalidad, básicos para nuestro estudio, se tratará de evidenciar dos aspectos, primero, el que hace referencia a la naturaleza representacional e intencional de las redes consiguientes a partir de las aportaciones del conocimiento socialmente distribuido y de la actividad situada y, el segundo, el que hace referencia a la naturaleza coordinada, sincronizada y compleja del rodaje como un equipo coordinado o una comunidad de práctica. En el entorno y proceso cinematográfico, como en cualquier otro, se da un intercambio de recursos representacionales y una dinámica propia del campo cinematográfico.

En un segundo apartado, se pondrá de manifiesto la conveniencia del Análisis de Redes Sociales, ARS, para representar las características de complejidad, coordinación y sincronización del proceso de rodaje. Mediante análisis básicos de centralidad y de agrupación, se identificarán y definirán los agentes que poseen el control local del proceso, es decir, aquellos que aseguran las condiciones de posibilidad reales del rodaje. Sobre una base intencional, las redes sociales elaboradas nos dan una representación externa de las condiciones y resultados reales bajo la forma y tipo de una red técnico-funcional. En concreto, trabajaremos con tres medidas de centralidad, grado, cercanía y proximidad, y tres de agrupación, cliques, n-clan y k-plex, que nos darán información acerca del control local existente y de las características funcionales y comunicativas del proceso de rodaje. En definitiva, nos introduciremos así, justificándolo, en uno de los aspectos de la dimensión social de la cognición como es la consideración de la red como una representación observada del conocimiento situado y distribuido en tanto que resultado de un proceso de comunicación y de manipulación instrumental. 
Brevemente, en un tercer apartado, se darán algunos elementos acerca de los límites del proceso, así como de la segunda intencionalidad del director, como agente central, que son necesarios para entender las conclusiones del análisis.

Finalmente, en un cuarto apartado de conclusiones elaborarán dos conceptos que son explicativos del rodaje cinematográfico y, especialmente, del funcionamiento real de proceso de representación como proceso de trabajo interactivo y social. En concreto, se definirá la paradoja del director y la dualidad cinematográfica. A partir de estos conceptos, se explicará la necesidad de ir más allá de la representación por redes, para explicar su capacidad de creatividad y de representación, como consecuencia del campo o mercado cinematográfico. Se considera que las aportaciones del conocimiento socialmente distribuido y de la actividad situada, así como la propia metodología de redes, ignoran muchas veces el factor del poder social, de la desigualdad de partida de los recursos representativos. Para llegar a un análisis sociológico del rodaje cinematográfico, por lo tanto, es necesario un intento de síntesis como el que se realiza en este artículo para entender el proceso real de representación.

\section{El rodaje cinematográfico: la necesidad del entorno, la intencionalidad y el proceso de representación}

¿Por qué nos parece pertinente para el análisis e interpretación de un proceso social en situación y más concretamente, como es el caso, el de un rodaje cinematográfico, usar como metodología la observación directa, de carácter casietnográfico, de caso, en sus propias condiciones de realización y no optar por centrarse solamente en entrevistas a los agentes implicados en el rodaje o en documentos secundarios o artículos de expertos acerca del funcionamiento de la industria cinematográfica?

En sociología, y extensivamente en las ciencias sociales, la comprensión y/o explicación de los hechos depende, evidentemente, de sus estructuras y dinámicas internas, pero éstas son inseparables del entorno y de los contextos de su realización. Es decir, aún tratándose del análisis de macro-fenómenos y de la aplicación de metodologías cuantitativas, siempre estamos estudiando comportamientos situacionales, prácticos y contextualizados, ya que la práctica tiene una lógica que no es la de la lógica (Bourdieu en Flyubjerg: 2001, 38). Por ello, la observación empírica y situacional de comportamientos es la manera menos arbitraria y válida de operativizar en indicadores los conceptos elaborados teóricamente. En este sentido, Geertz (1974) afirma que el entorno es una fuerza disciplinaria poderosa, asertiva, exigente y hasta coercitiva. En definitiva, optando por la observación directa, durante el mayor lapso de tiempo posible -que en el 
caso del material recopilado para este artículo es demasiado poco, por falta de recursos temporales, una de las razones existentes para hablar de observación casi-etnográfica, y no etnográfica-, la misma actividad observada pide realismo al científico. Es decir, una correcta contemplación de la realidad empírica en la situación de su realización permite la recopilación de mayor material a indexar y codificar, y también una compresión más inmediata y directa, pragmática, émica y empática de los fenómenos observados, contemplación no habitual en el quehacer sociológico.

En el caso del rodaje cinematográfico, además, nos encontramos ante un fenómeno sincrético de cognición social. Es decir, se trata de una producción colectiva con una determinada estructura social que han de ser ambas observadas en el continuum comunicativo entre los miembros y artefactos del equipo cinematográfico. Más adelante veremos cuáles son los elementos de esta estructura social, es decir, los factores sociales que definen la distribución del conocimiento necesario para realizar el trabajo en equipo, así como los otros elementos que regulan el intercambio comunicativo e instrumental entre los trabajadores y las trabajadoras del rodaje. De momento, la naturaleza distribuida de las comunicaciones e interacciones entre los miembros del rodaje da al proceso observado una característica de complejidad y una aparente atmósfera de caos: la falta de unas pautas estrictas, lógicas, de organización de la actividad (Valsiner \& Van der Veer, $1995,393)$. La necesidad de poner orden a lo observado o de comprender el caos y de encontrar sistemas conceptuales explicativos, nos obliga a partir del contenido y del significado de las interacciones observadas. Como veremos al hablar de la intencionalidad de los agentes, el método cualitativo de observación permite, más que cualquier otro método de investigación social, ver la contribución de uno u otro individuo (o miembro del equipo cinematográfico) siempre en relación con la actividad completa (el rodaje), sin extraer tal contribución de su entorno (Rogoff a Valsiner y Van der Veer: 1995, 395). Dado el bajo grado de estandarización y de sistematización de las prácticas del rodaje, no podemos estudiar tales contribuciones, es decir, la participación e interacción de los agentes en la filmación de la película, de forma aislada, como unidades reducidas de análisis, o como producto de un conocimiento de receta, sino que debemos partir del resultado de situaciones sociales observadas y concretas y además consideradas como unidad global de análisis según las orientaciones del conocimiento socialmente distribuido.

Además, a tal variabilidad del proceso interactivo, que exige la observación del comportamiento actual de los agentes -en oposición al comportamiento narrado o externamente documentado-, se le añade la necesidad de contar con un sólido aparato conceptual previo o en proceso de elaboración en el análisis, ya que las 
teorías no provienen nunca de los hechos (Canguilhem a Bourdieu, Chamboredon, Passeron: 2000, 285). Esta afirmación puede 'traducirse', desde las orientaciones teóricas aquí adoptadas, en que las teorías no son parte o residen en los hechos, aunque se produzcan a partir de la práctica como representación. Si bien el material empírico para el análisis de redes surge de la observación con cámara de vídeo de un rodaje real de una escena cinematográfica en la ciudad de Barcelona, solamente es analizable a partir de las definiciones conceptuales y marcos teóricos que previamente o en el proceso de análisis iremos construyendo y que consideraremos pertinentes.

\section{EI rodaje cinematográfico: un proceso de representación}

Nuestro objeto de estudio, por lo tanto, es la observación de un rodaje cinematográfico, entendido como un proceso social colectivo comprendido por una secuencia de intencionalidades más o menos imaginativas, es decir, con un grado variable de capacidad de intervención e interacción en la producción de representaciones.

La primera exigencia, dada la complejidad del proceso y su evidente extensión espacial y temporal, consiste en delimitar, como unidad de análisis y observación elegidos, las prácticas, interacciones, agentes e instrumentos y tareas del rodaje y su organización, complejidad incrementada por las circunstancias pragmáticosociales que puedan surgir en el proceso y debido al entorno y contexto de la producción.

Para llegar a las consecuencias productivas de la capacidad intencional de los agentes, debemos tener en cuenta que el conocimiento en la práctica cotidiana se distribuye, se esparce y de divide entre la mente, el cuerpo, la actividad y los entornos organizados socialmente y que incluyen otros agentes (intencionales) (Lave a Barab et al: 2001, 67). Uno no puede reducir los procesos cognitivos a simples sistemas físicos de entrada como son los mecanismos de percepción, memoria o atención (Searle: 2004). Gracias al desarrollo del modelo lingüístico inferencial y a los descubrimientos de la psicología evolucionista, la mente se puede describir ya no como instrumento autónomo, sino como una entidad dependiente, enteramente social (Vygotsky: 1962, Sperber \& Wilson: 1995).

Así, toda actividad cognitiva es una combinatoria necesaria entre representaciones internas (imágenes mentales y recuerdos), y externas (gráficos, cuadros, textos, ordenadores y otros instrumentos informativos). Dentro de esta perspectiva técnica y funcional, dicha dependencia instrumental pone de relieve cualquier tipo de manipulación instrumental o decisión cognitiva, hasta la más automática y simple. 
No obstante, el agente que trabaja no es tan sólo práctico, sino también intencional, con la capacidad mental de producir estados mentales que se refieren, o que son sobre, o que directamente son objetos y estados del mundo externos a ellos mismos (Searle: 2004). Es decir, la aplicación de mecanismos mentales de intencionalidad derivada, siguiendo a Searle (1983) nos capacitan, como agentes sociales, para formular deseos, creencias y representaciones a partir de las representaciones de los otros agentes que nos rodean. En definitiva, el mecanismo de la intencionalidad es el mecanismo evolutivo que nos garantiza nuestro devenir social, en la medida que nos lleva a la interacción para maximizar nuestra satisfacción comunicativa y aumentar nuestro bagaje representacional.

Dicho esto, debemos tener en cuenta que, desde una perspectiva sociológica como la que adoptamos, no podemos conocer los estados mentales de los agentes, es decir, sus representaciones derivadas internas. Así que nuestro objeto de estudio no es la descripción de tales mecanismos mentales, que dejamos a la psicología evolutiva y cognitiva, sino la descripción de las relaciones comunicativas e instrumentales que construyen la naturaleza interactiva del rodaje cinematográfico. Nuestra intención es aprovechar tales aportaciones teóricas para fundamentar la naturaleza cognitiva del agente que trabaja, huyendo así de las visiones atomistas de la acción social y tener así en cuenta la complejidad de toda actividad productiva. Observando la realidad y representándola, como veremos en la configuración de las redes sociocéntricas, pensamos el mundo de forma objetiva en la medida que podemos pensarlo como intersubjetivo (Pettit: 1986). Paralelamente, los miembros del equipo de rodaje aplican los mecanismos cognitivos de percepción, de atención, o bien las categorías lingüísticas, partiendo de la interacción social, en la que la existencia de otras mentes comunes son necesarias (Pettit: 1986).

En definitiva, asumiendo la naturaleza intencional de los agentes, definiremos el rodaje como un proceso situacional, que denominamos en su conjunto "entorno", conformado y compuesto por una determinada red técnico-funcional de agentes intencionales y de recursos representacionales con distintos niveles de complejidad, coordinación, sincronización y dependencia instrumental, e inscrito en unos contextos y campos específicos. De esta forma, el proceso de representación es social en tanto que interactivo e intencional. Más allá de estas dos dimensiones, que representaremos por redes técnico-funcionales, existe una tercera que establece la importancia de las relaciones sociales, y que se basa en el análisis de los recursos intercambiados en el proceso. 
En esta definición del rodaje como proceso de representación, introducimos así el término "recurso representacional". Éstos constituyen el conjunto de recursos de todo tipo, utilitarios, económicos, de signo cognitivo y los puramente sociales, que se intercambian los agentes en el conjunto de tareas del proceso. En el momento del análisis empírico por redes llenaremos de contenido los recursos del proceso de rodaje. No obstante, es importante decir aquí que estos recursos, poseídos por los agentes, constituyen, además, un factor social clave en el proceso de representación cinematográfico. Así, además de ser un proceso interactivo el rodaje se sitúa y dinamiza en un campo cinematográfico, es decir, es parte del espacio social, siguiendo a Bourdieu (1998, 1994, 1979, y Wacquant: 1994), delimitado por una determinada estructura o distribución de capitales sociales de diferente naturaleza. Estos capitales sociales los concebimos como el conjunto de cualidades sociales, actividades o recursos representacionales (materiales, lingüísticos, relacionales o económicos) con una pertinencia social y un valor que provendría y constituiría, justamente una vez apropiado por el agente, la posición o capital social del agente que lo posee en el campo. Sin entrar de lleno en el planteamiento de espacio, capital y habitus de Bourdieu, sí que introducimos aquí la necesidad de considerar concretamente el rodaje cinematográfico como parte de un doble mercado cultural y económico, es decir, como parte de un dominio artístico y de una industria cinematográfica. Las interacciones que observamos entonces son la expresión de los mecanismos de la doxa del campo, que califican a unos capitales de dominantes, y con un mayor rendimiento económico y simbólico dentro del campo correspondiente, y otros como dominados, según volumen y estructura.

Volviendo al rodaje cinematográfico, los recursos representacionales intercambiados en el proceso son concreciones empíricas, mensurables y comparables, de la estructura de capitales del campo cinematográfico, al mismo tiempo que cambian o estabilizan el estado de dichos capitales en el campo. Si, como venimos diciendo, el rodaje cinematográfico, ejemplo de proceso técnicoartístico, surge del conjunto de decisiones y de acciones de los agentes intencionales, debemos tener en cuenta, como lo hace Bourdieu cuando define los principios de la distinción, de homología y de autonomía, que tales decisiones y prácticas se basan en las posiciones y estatus, y por tanto en las relaciones con otros, que los agentes tienen, en cuanto poseedores de diferentes formas de capital.

Las dos posiciones básicas que, como 'lugares sociales' de posesión de capital dentro del campo de un rodaje cinematográfico, consideramos son: 
Agentes o posiciones centrales: recursos representacionales dominantes propios a procesos de decisión centrales.

Agentes o posiciones periféricos: recursos representacionales dominados: procesos de decisión subordinados.

I lustración 1. Los agentes participantes

Como hemos visto brevemente, la capacidad de decisión depende de la posición del agente en el campo, y de su posesión de capital social dominante. En consecuencia, la capacidad para representar y participar en la secuencia de intencionalidades y en su concreción en representaciones, que organizan la producción cinematográfica, constituyen un factor y resultado social de dominación. En definitiva, si el pensamiento es libertad en relación con lo que uno hace (Flyubjerg: 2001, 127), tenemos que hablar más bien de un pensamiento libremente condicionado, por ser potencialmente libre, pero limitado y conectado realmente a las factores sociales de dominación.

Dado que el concepto fundamental de la ciencia social es el poder (Russell a Flyubjerg: 2001, 88), el objetivo final del análisis de redes de un rodaje no será tan sólo la descripción de una secuencia de interacciones, intencionalidades y sus representaciones más o menos artísticas, sino también el análisis de relaciones de dominación entre agentes con determinados recursos dominantes $\mathrm{y}$, otros, con recursos dominados.

Se trata, entonces, de la observación de un proceso social aunque sea de un acontecimiento o proceso y, por tanto un problema situacional. En efecto, tal como vienen diciendo los teóricos del CSD (Barab, Hay, Yamagata-Lynch: 2001; Bardram: 2000; Cicourel: 2002; Engeström i Midleton: 1996; Giere i Moffat: 2003; Hutchins: 1995; Jonassen i Reeves: 1993; Zhang i Norman: 1994) el conocimiento puesto en juego, necesario para realizar por ejemplo, una escena cinematográfica, no consiste sólo en una formulación o exposición racional, deductiva y sólo internalizada por el individuo o en la mente. Al contrario, todo conocimiento surge y al mismo tiempo promueve, la confrontación interactiva entre agentes que actúan en un entorno dado, realidad generativa eminentemente social porque es distribuida, es relacional y procura la diferenciación entre los que trabajan. Tal como afirma Dreyfus (1993), el conocimiento aplicado en el trabajo de expertos en la vida real es rápido, holístico, heurístico y también visual. De este modo, la observación del intercambio de recursos y de interacciones, a través de la plasmación de las representaciones externas de los agentes, es una forma de llegar a este tipo de conocimiento aplicado y pragmático que se aleja de concepciones 
mentalistas e individualistas, o de otras holistas pero no suficientemente intencionales.

En definitiva, si hablamos de proceso de representación, es porque las representaciones informativas, que conforman el conocimiento distribuido entre los agentes del proceso de trabajo, constituyen un conjunto coherente de representaciones que están articuladas y estructuradas a partir de la lógica y de las pautas de los procesos del sistema interactivo y cognitivo (Lozares: 2005).

\section{El rodaje: conocimiento socialmente distribuido y estrategias de dominación social}

Siguiendo en la perspectiva del conocimiento socialmente distribuido, tenemos que dar un par de características más de la naturaleza de la producción cognitiva en el entorno de trabajo, que nos guiará en la observación del rodaje cinematográfico. En efecto, a partir del conocimiento socialmente distribuido el entorno profesional del proceso es contemplado desde la organización social del trabajo. La dimensión colectiva del proceso de representación se acompaña de una necesidad de coordinación entre los agentes, ya que dependen unos de otros para solucionar los problemas que surjan y así avanzar hacía el producto final.

La cooperación y la coordinación temporal, por lo tanto, son claves en el rodaje cinematográfico. Tres son los tipos de dependencia interactiva que existen entre los agentes que trabajan: primero, los prerrequisitos de la acción, un proceso de preproducción que en el rodaje es clave para decidir la localización, los cargos de los agentes y los horarios de trabajo; segundo, la simultaneidad de actividades, que implica el desarrollo de tareas en paralelo, en un mismo momento temporal y espacial; y, tercero, los recursos compartidos requeridos para las múltiples actividades (Bardram, 2000). Esta dependencia instrumental se añade a la interdependencia entre los agentes por la distribución de conocimientos existentes, representada por los recursos representacionales, de tipo signo cognitivo (verbal o no) que describiremos en las redes.

En concreto, el rodaje cinematográfico incluye un alto grado de tecnificación y de profesionalización, característica central en un entorno profesional y que, si bien puede aumentar el nivel de autonomía de los agentes aumentando su capacidad de decisión, también pueden convertirse en un elemento de constricción. No obstante, siguiendo el optimismo de Benjamin $(1983,61)$, la reproducibilidad técnica emancipa la obra de arte, por primera vez en la historia del mundo, de su existencia parasitaria dentro de la esfera del ritual... de la ilusión de su autonomía. Además de la presencia de recursos instrumentales-técnicos, la frase de Benjamin 
nos incita ya a tener en cuenta tales recursos como incidentes en la elaboración del producto final, la escena cinematográfica.

Volviendo a lo que decíamos más arriba, nos centramos así en los recursos disponibles para todos los agentes, sean dominados o dominantes, para llegar a la distribución real de conocimiento que no dependerá solamente, como veremos, de la necesidad local de coordinación, sino que seguirá la confrontación de estrategias de distinción del campo social cinematográfico.

Daremos cuenta a continuación de la recogida de material empírico mediante la observación casi-etnográfica con cámara de vídeo, lo que nos dará un conocimiento local, o descripción densa, en palabras de Geertz (1983), de las formas de relación y de actuación de los agentes en la situación de producción cinematográfica. Una vez definida la red técnico-funcional en la que se basa la producción de conocimiento cinematográfico, daremos un paso más y, a través del prisma de los recursos representacionales, se explicaran dos mecanismos de poder ejercidos por la macro-organización del campo cinematográfico.

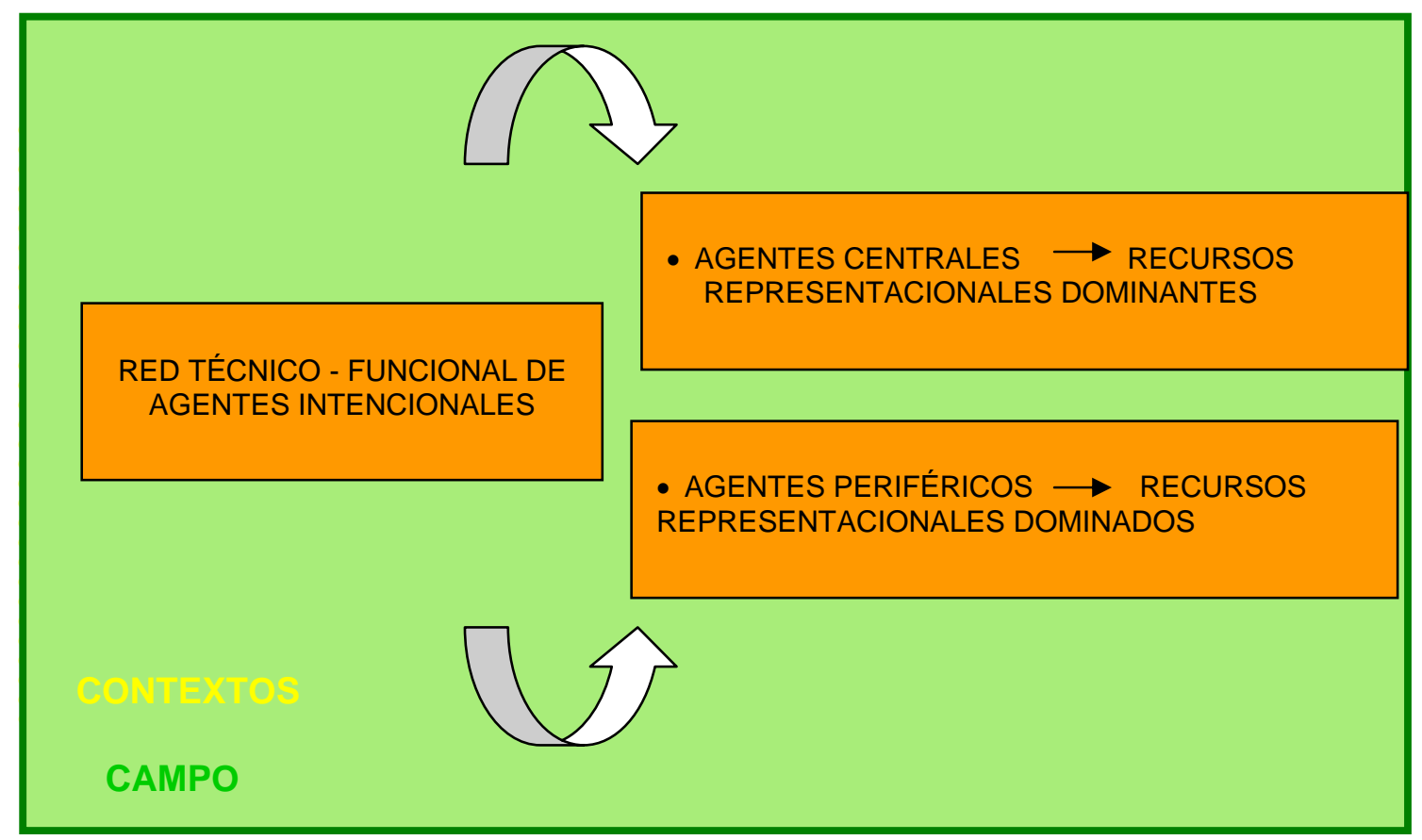

Ilustración 2. El proceso de representación cinematográfico: En el gráfico vemos el proceso de representación como proceso inscrito en un campo social, con sus contextos correspondientes y los recursos representacionales distribuidos de manera no- uniforme entre los agentes. Precisamente el intercambio de recursos y sus interacciones entre agentes constituyen la red técnico-funcional, observable en la producción del resultado final, la escena cinematográfica. 


\section{El rodaje según redes sociales: coordinación, sincronización, complejidad y control local del conocimiento situado}

Las redes sociales que representan la red técnico-funcional del rodaje constituyen nuestro mejor argumento, si aceptamos que el mejor argumento es un concepto empírico (Flyubjerg: 2001, 93). Primero, nos permiten descubrir las características interactivas del proceso que hemos apuntado en el primer apartado teórico $y$, segundo, delimitan la densa red de relaciones que constituyen la dimensión de poder de toda relación productiva. Así, sin olvidar los factores estructurales de clase, que agrupan y clasifican socialmente los agentes, partiremos de las aproximaciones de Bourdieu apuntadas más arriba para descubrir la dominación y 'sumisión' local a nivel comunicativo y representacional. Es decir, nos interesa ver cuál es el grado de participación de los agentes en la producción de conocimiento distribuido y qué posibles mecanismos de poder, provenientes del mercado artístico, pueden influenciar o configurar las relaciones laborales entre los miembros del equipo cinematográfico.

El uso de las redes sociales que a continuación se presentan es, en una primera instancia, de tipo instrumental y 'transustivamente' cognitivas y sociales: si el análisis reticular es interesante, es gracias a su flexibilidad y heterogeneidad (Luz González y Molina: 2002). Es decir, la red permite representar todo tipo de conceptos, ya sean macros o micros: en nuestro caso, representaremos recursos representacionales que conectan socialmente los agentes del rodaje, por lo que se trabajará con un concepto intermedio, ya que es parte del campo social (macro) y, a la vez, es producto del intercambio intencional entre los agentes. A esa flexibilidad conceptual se le suma la heterogeneidad que atorga a los usuarios de esta metodología la posibilidad de partir de puntos de vista teóricos diferenciados. Así, en el caso que nos ocupa, se parte de la aproximación del conocimiento socialmente distribuido, pero también de las explicaciones intencionales de Searle (1983) y de la teoría de la reproducción de Bourdieu (1979, 1994, 1998), para explicar la posición de los agentes en la red técnico-funcional del rodaje cinematográfico.

Recurrimos al ARS usando el programa UCINET para el análisis de la interacciones/relaciones habidas provenientes de las observaciones hechas en el rodaje de un telefilme, la semana del 13 al 21 de mayo de 2005, en un hospital público de Barcelona. En concreto, la observadora estuvo tomando notas y filmando con una càmara de vídeo con trípode, el conjunto de actividades de rodaje de un equipo formado por 33 personas, distribuidas en cargos profesionales según el siguiente esquema: 


\begin{tabular}{|c|c|}
\hline CARGO & NODO \\
\hline \multicolumn{2}{|l|}{ REALIZACIÓN } \\
\hline Director & director \\
\hline Ayudante de dirección & ajudant \\
\hline 2n Ayudante & ajuda2 \\
\hline 3r Ayudante & ajuda3 \\
\hline Meritoria de dirección & ajuda4 \\
\hline \multicolumn{2}{|l|}{ PRODUCCIÓN } \\
\hline Productor ejecutivo & productor \\
\hline Directora de & direprod \\
\hline producción & prod2 \\
\hline Jefe de producción & prod3 \\
\hline Jefe de producción2 & ajudaprod2 \\
\hline Auxiliar de producción & ajudaprod3 \\
\hline $\begin{array}{l}\text { Auxiliar de producción } \\
\text { Runner }\end{array}$ & runner \\
\hline \multicolumn{2}{|l|}{ FOTOGRAFIA } \\
\hline Director de fotografía & cámara \\
\hline foquista & focus \\
\hline Auxiliar cámara & focus2 \\
\hline Técnica de vídeo & vídeo \\
\hline
\end{tabular}

\begin{tabular}{|c|c|}
\hline CARGO & NODO \\
\hline DECORADOS/ATREZZO & \\
\hline $\begin{array}{l}\text { Dirección artística } \\
\text { Ayudante de decoración } \\
\text { Atrezzista } \\
\text { Jefe de construcción } \\
\text { Constructor } \\
\text { Figurinista }\end{array}$ & $\begin{array}{l}\text { capdeco } \\
\text { atrezzo2 } \\
\text { atrezzo3 } \\
\text { capatrezzo } \\
\text { deco2 } \\
\text { figurinista }\end{array}$ \\
\hline MAQUILLAJE & \\
\hline $\begin{array}{l}\text { Maquilladora } \\
\text { Peluquera }\end{array}$ & $\begin{array}{l}\text { maquilla } \\
\text { maquilla2 }\end{array}$ \\
\hline ELÉCTRICOS & \\
\hline $\begin{array}{l}\text { Jefe de eléctricos } \\
\text { Eléctrico } \\
\text { Eléctrico }\end{array}$ & $\begin{array}{l}\text { capelectri } \\
\text { electri2 } \\
\text { electri3 }\end{array}$ \\
\hline SONIDO & \\
\hline $\begin{array}{l}\text { Jefe de sonido } \\
\text { Ayudante de sonido }\end{array}$ & $\begin{array}{l}\text { capso } \\
\text { so2 }\end{array}$ \\
\hline
\end{tabular}

Tabla 1. Lista de cargos profesionales del rodaje

Los cargos de las personas son un producto contextual, en el sentido que son el resultado de reuniones de preproducción, anteriores al rodaje, dirigidas por el productor ejecutivo, que normalmente no se encuentra en el rodaje. Aunque no hablaremos más de ello en este artículo, sí que diremos que existe un segundo grupo de factores sociales, además de los recursos representacionales, que inciden en las estrategias de dominación del rodaje, que llamamos contextos. Estos, que no se deben confundir con el entorno o con la situación del proceso observado, son agentes o recursos que son parte del campo que inciden en el rodaje, pero que no forman parte estrictamente del entorno. Concretamente, el productor ejecutivo y el guionista son ejemplos de dicho tipo de agentes que inciden con su intencionalidad en el proceso, a través de recursos como hoja de cargos (como la que tenemos más arriba) y/o los horarios de trabajo o el guión, en el caso del guionista.

Una vez hecho este apunte conceptual, diremos algo acerca de la composición por género del equipo; dado que es un factor social clave del espacio social, tendría que ser tratado en un apartado aparte. No obstante, sí que podemos decir que la distribución profesional sigue la tendencia del espacio social y la segmentación correspondiente del mercado de trabajo. Es decir, existe una mayoría de hombres en las áreas con un mayor poder de decisión en el proceso, como veremos con el análisis (realización y producción sumados) y en los sectores profesionales tradicionalmente masculinizados (eléctricos y sonido), mientras que las mujeres son mayoría en los sectores tradicionalmente feminizados (maquillaje y peluquería), y en las posiciones de ayudantías de cualquier área. Por lo que a edad 
respecta, la mayoría de técnicos son menores de 40 años, y los de más edad son el director, el director de fotografía, el ayudante de dirección y la jefa de producción.

\section{Las tres redes sociocéntricas elaboradas}

Retornado ya a las tres redes creadas, se parte del material empírico recogido en notas de campo y por filmación del rodaje de una sola escena cinematográfica, el 16 de mayo, de 9 a 12 de la mañana. Se rodó en exteriores durante 3 horas y se hicieron 5 tomas según la decisión del director. Los límites del proceso se marcaron siguiendo los siguientes criterios: una unidad comunicativa entre los agentes; una relativa estabilidad y equilibrio del producto resultante, es decir, la escena rodada; una unidad espacial y temporal; la presencia invariable de los agentes participantes; y la existencia de unos agentes iniciadores y finalizadores del rodaje. Precisamente, en el apartado siguiente nos extenderemos con la quinta característica expuesta, ya que nos dará más información acerca de la organización real del equipo de rodaje.

Del conjunto de relaciones observadas hemos hecho una clasificación en tres redes generadoras. La primera, es una red comunicacional básica, la segunda comunicacional informacional, y la tercera, instrumental. Las tres están formadas por las relaciones de intercambio de recursos, por lo que los nodos son los agentes del rodaje, con la excepción de la tercera, que también incluye recursos, como veremos. Se simbolizan con triángulos los agentes de género masculino y con círculos los de género femenino. Además, el color diferencia los nodos por cargos, que hemos dividido en nueve grupos: realización, producción, fotografía, atrezzo, maquillaje, eléctricos, actores, script y sonido.

A partir del modelo teórico que apuntábamos al empezar este artículo, las tres redes dan cuenta de la red técnico-funcional, ya que recogen la totalidad de interacciones entre los trabajadores y las trabajadoras primero; las interacciones profesionales-funcionales segundo; y las manipulaciones instrumentales tercero. Así, recogemos información acerca del tipo de recursos representacionales intercambiados o no, y del tipo de tomas de decisión de los agentes que participan en el rodaje. A continuación mostramos las tres redes, con su correspondiente definición:

1) Red comunicacional básica. Recoge la totalidad de intercambios verbales, formales e informales, entre agentes del proceso de representación, dentro del intercambio de recursos de signo cognitivo. Es decir, a partir del material observado en la filmación y directamente, se recoge toda interacción verbal, independientemente de su contenido, entre los miembros del equipo, durante la 
filmación de las tomas, y durante el tiempo transicional de preparación entre toma y toma.

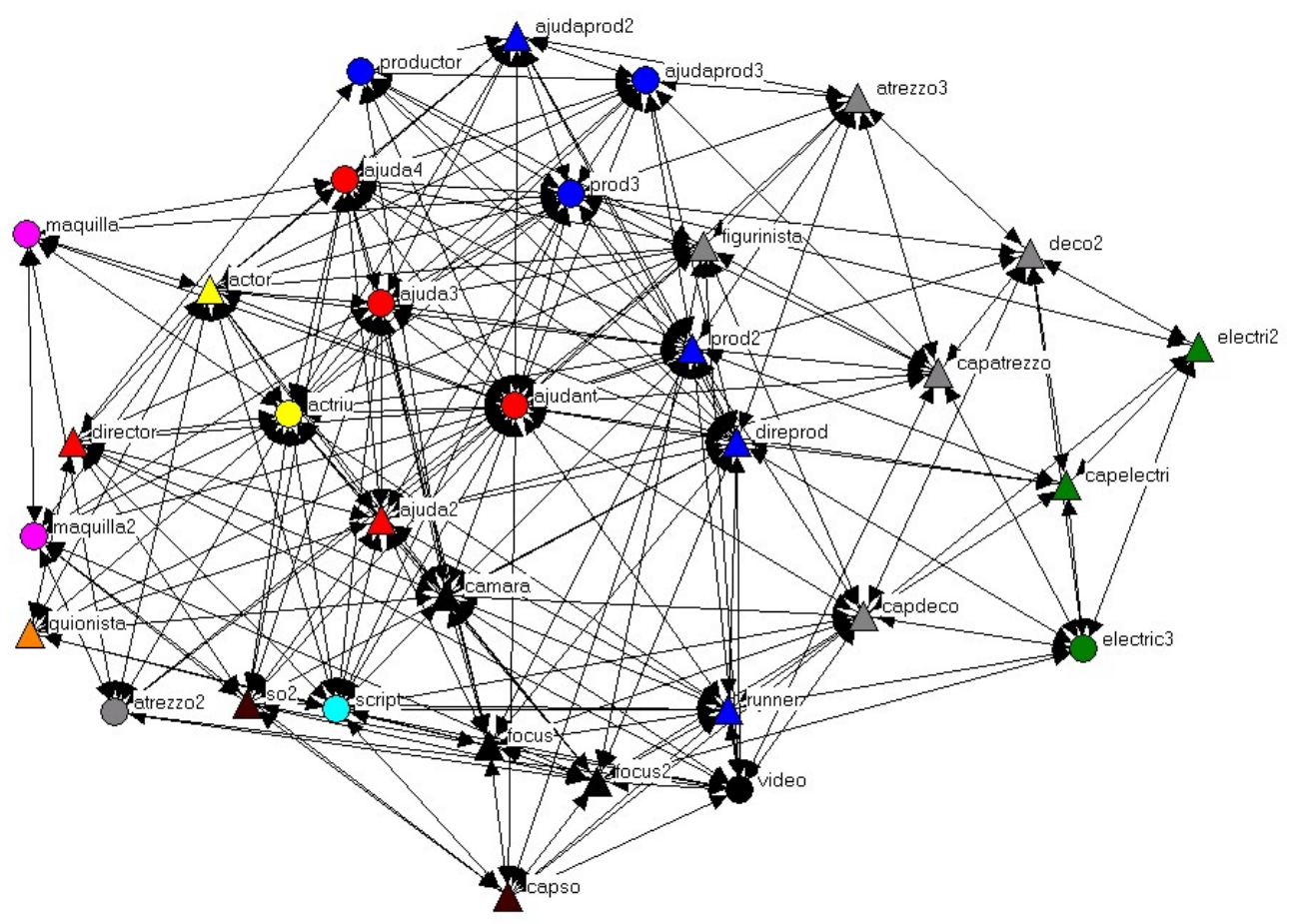

\section{Leyenda}

Formas: GÉNERO

Triángulo: Masculino

Círculo: Femenino

Colores: CARGOS de los agentes participantes en el proceso de rodaje

\begin{tabular}{|c|c|c|c|c|c|c|c|}
\hline & & & & & & & \\
\hline Realización & Producción & Fotografía & Atrezzo & Maquillaje & Eléctricos & Actores & Script \\
\hline $\begin{array}{l}\text { Director } \\
\text { Ajudant } \\
\text { ajuda2 } \\
\text { ajuda3 } \\
\text { ajuda4 }\end{array}$ & $\begin{array}{l}\text { productor } \\
\text { direprod } \\
\text { prod2 } \\
\text { prod3 } \\
\text { ajudaprod2 } \\
\text { ajudaprod3 } \\
\text { runner }\end{array}$ & $\begin{array}{l}\text { càmara } \\
\text { focus } \\
\text { focus2 } \\
\text { vídeo }\end{array}$ & $\begin{array}{l}\text { capdeco } \\
\text { atrezzo2 } \\
\text { atrezzo3 } \\
\text { capatrezzo } \\
\text { deco2 } \\
\text { figurinista }\end{array}$ & $\begin{array}{l}\text { Maquilla } \\
\text { maquilla2 }\end{array}$ & $\begin{array}{l}\text { capelectri } \\
\text { electri2 } \\
\text { electric3 }\end{array}$ & $\begin{array}{l}\text { actor } \\
\text { actriu }\end{array}$ & script \\
\hline SONIDO & & & & & & & \\
\hline $\begin{array}{l}\text { Capso } \\
\text { So2 }\end{array}$ & & & & & & & \\
\hline
\end{tabular}

I lustración 3. Red sociocéntrica de los agentes participantes. Elaboración propia con UCINET

2) Red comunicacional informacional. Recoge los intercambios verbales profesionales, durante los cuales los agentes intercambian recursos de signo cognitivo verbales necesarios para el desarrollo del rodaje. Así, se incluyen solamente las interacciones informativas, el contenido de las cuales sea imprescindible para la realización del producto final, la escena cinematográfica. 


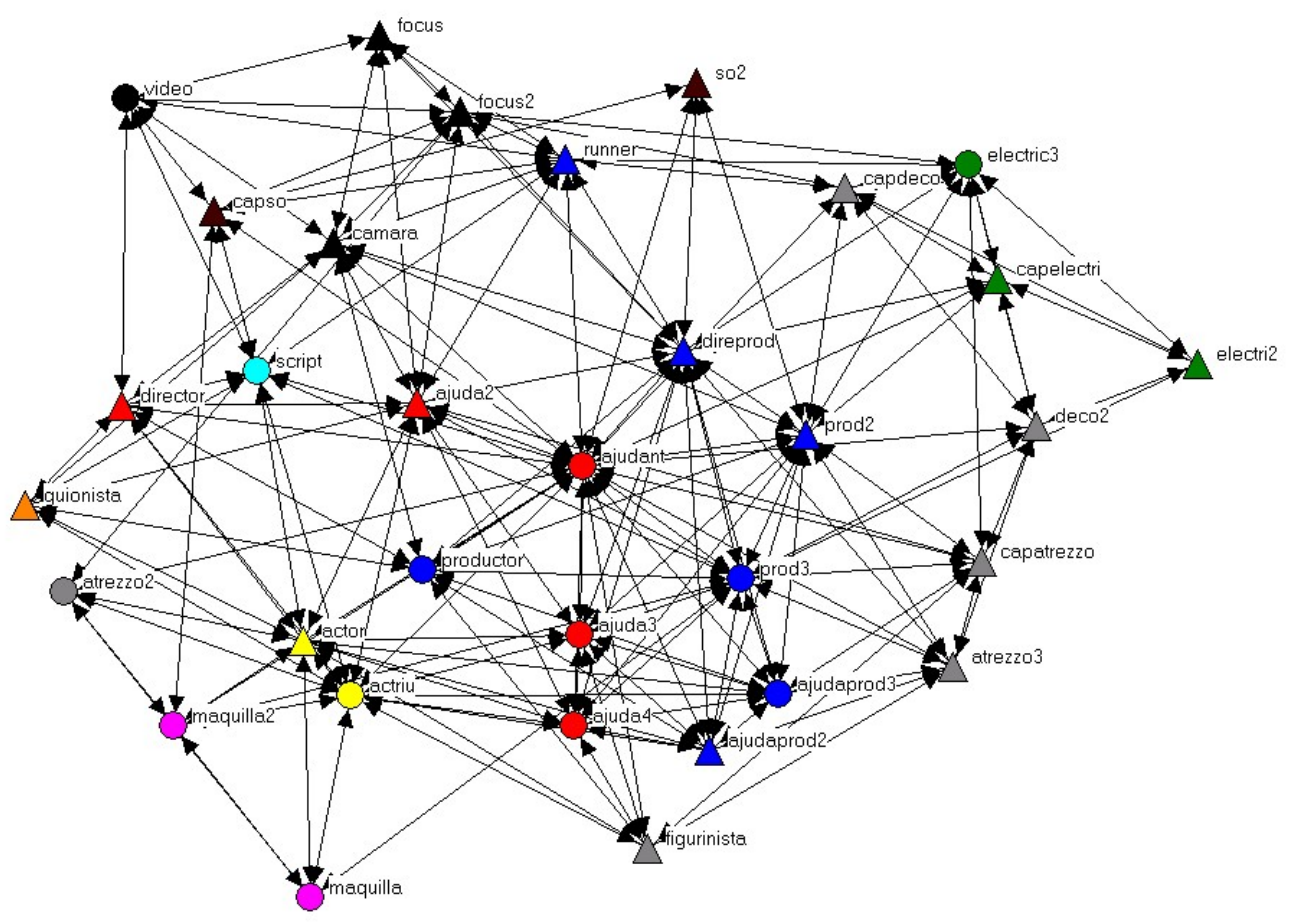

\section{Leyenda:}

Formas: GÉNERO

Triángulo: Masculino

Círculo: Femenino

Colores: CARGOS de los agentes participantes en el proceso de rodaje

\begin{tabular}{|c|c|c|c|c|c|c|c|}
\hline \\
\hline Realización & Producción & Fotografía & Atrezzo & Maquillaje & Eléctricos & Actores & Script \\
\hline $\begin{array}{l}\text { Director } \\
\text { Ajudant } \\
\text { ajuda2 } \\
\text { ajuda3 } \\
\text { ajuda4 }\end{array}$ & $\begin{array}{l}\text { productor } \\
\text { direprod } \\
\text { prod2 } \\
\text { prod3 } \\
\text { ajudaprod2 } \\
\text { ajudaprod3 } \\
\text { runner }\end{array}$ & $\begin{array}{l}\text { càmara } \\
\text { focus } \\
\text { focus2 } \\
\text { vídeo }\end{array}$ & $\begin{array}{l}\text { capdeco } \\
\text { atrezzo2 } \\
\text { atrezzo3 } \\
\text { capatrezzo } \\
\text { deco2 } \\
\text { figurinista }\end{array}$ & $\begin{array}{l}\text { Maquilla } \\
\text { maquilla2 }\end{array}$ & $\begin{array}{l}\text { capelectri } \\
\text { electri2 } \\
\text { electric3 }\end{array}$ & $\begin{array}{l}\text { actor } \\
\text { actriu }\end{array}$ & script \\
\hline \multicolumn{8}{|l|}{ SONIDO } \\
\hline $\begin{array}{l}\text { Capso } \\
\text { So2 } \\
\end{array}$ & & & & & & & \\
\hline
\end{tabular}

I lustración 4. Red sociocéntrica de los agentes participantes. Elaboración propia con UCINET.

3) Red instrumental. A diferencia de las precedentes, recoge la relación instrumental de los agentes, es decir, las interacciones entre los agentes participantes en el rodaje y los recursos utilitarios, económicos y de signo cognitivo no verbal. Así, se ejemplifica la dependencia instrumental de los agentes respecto de los recursos instrumentales y, sobretodo, técnicos, tan presentes en el entorno. Se trata de una red de naturaleza mixta, porque recoge los 33 nodos-agente, y 18 nodos-recurso, clasificados en 6 grupos. Tal clasificación se ha realizado partiendo de la función de cada recurso en la red, y partiendo teóricamente de la importante dimensión instrumental del conocimiento producido en el rodaje. 
1) Recursos instrumentales-técnicos: Requieren un conocimiento específico por parte de quién los manipula, por lo que se trata de un tipo de recurso de signo cognitivo. Incluyen la cámara, el vídeo y las pantallas, el foco de la cámara, los micrófonos, y el resto de material eléctrico y lumínico, como los raíles, el transporte, etcétera. Según la función del agente a cargo, el recurso es más o menos técnico: en el caso de la cámara, el director de fotografía o cámara actúa de forma más creativa y artística, mientras que sus ayudantes aplican un tipo de conocimiento más técnico.

2) Recursos instrumentales-generales: Son recursos no específicos del rodaje, y que se utilizan en la vida cotidiana, sin constituir, por lo tanto, un recurso de signo cognitivo específico. Los constituyen las cintas adhesivas y aislantes con las que cargan los eléctricos, la carretilla con materiales eléctricos, la manta y la bolsa de vestuario para los actores, el cronómetro que utiliza la script para sincronizar los diálogos, el material de decoración para la ambientación del set... En el caso del material de atrezzo, se trata de un recurso especial, con una cierta carga de signocognitivo artístico, al ser un elemento que transforma un espacio físico, el set, en un espacio de ficción.

3) Recursos comunicativos: Los walkies y los móviles están presentes y son manipulados por absolutamente todos los agentes participantes: son recursos de signo cognitivo general en el caso del móvil, y específico en el caso del walkie.

4) Claqueta: Se trata de un recurso de alto significado de signo cognitivo artístico, ya que divide el proceso en sus fases (las tomas) de producción, y marca el principio y al final de éste.

5) Recursos de escritura: Los únicos recursos de signo cognitivo verbal escrito son manipulados por la script, en la corrección del guión, y por otros agentes de forma muy puntual.

6) Recursos utilitarios generales: Son recursos básicos, muy compartidos por los agentes, como la mesa de catering, con comida disponible en todo momento para los agentes participantes en el rodaje, y los cigarrillos, que son objeto de intercambio continuado. 


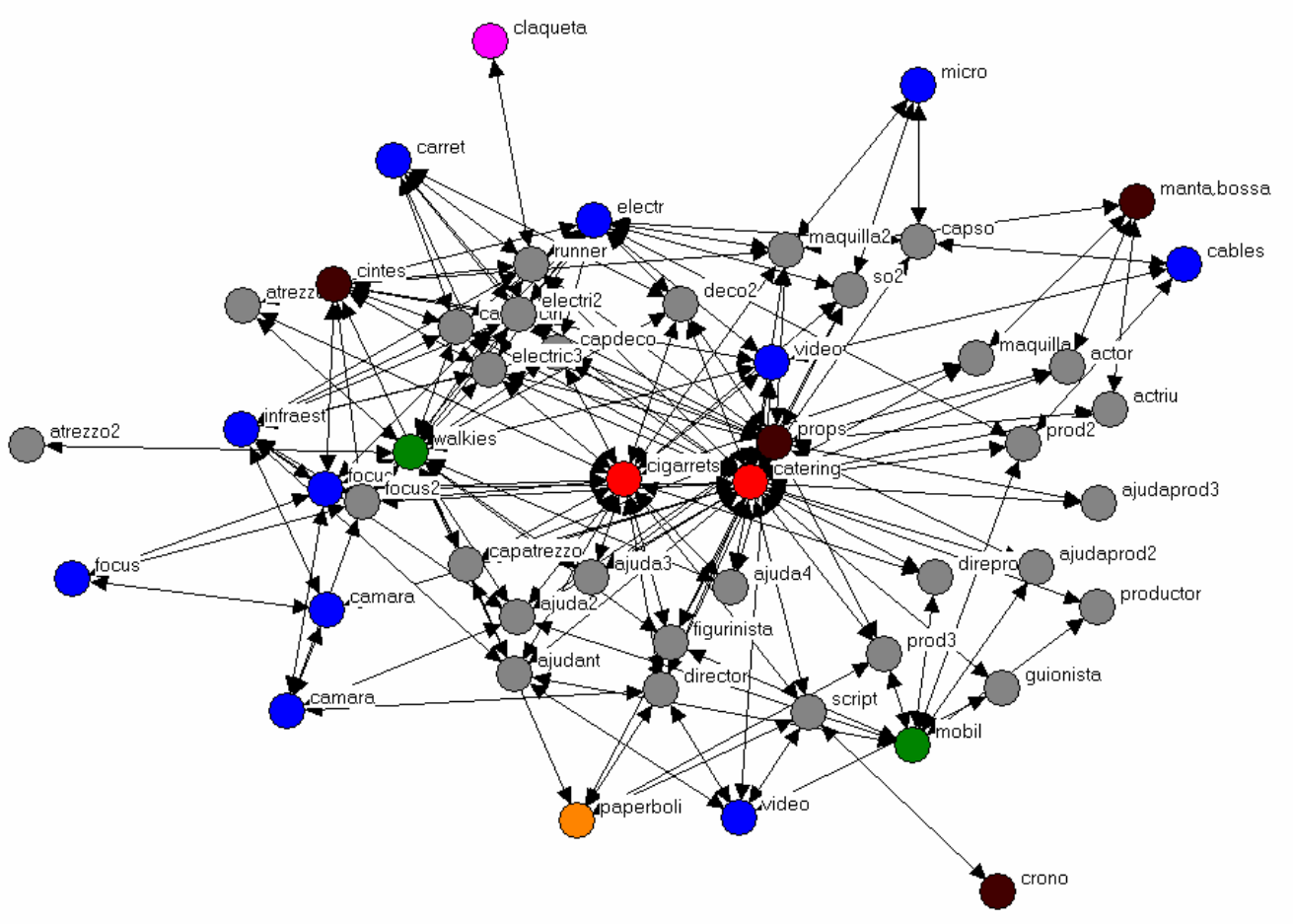

\section{Leyenda:}

AGENTES participantes y RECURSOS intercambiados en el proceso

Agentes participantes

Recursos utilitarios generales

Recursos instrumentales - técnicos

Recursos comunicativos

Recursos instrumentales generales

Claqueta

Recursos de escritura

I lustración 5. Red sociocéntrica de los recursos intercambiados. Elaboración propia con UCINET.

\section{El análisis reticular: medidas de centralidad y de agrupación}

Una vez presentadas estas tres redes sociocéntricas, pasaremos a exponer las medidas aplicadas (mediante el programa informático UCINET): la centralidad de rango y el índice de poder de Bonacich (A), la centralidad de proximidad (B), la centralidad y el flujo de intermediación (C) y un conjunto de medidas de agrupación (D). Como expondremos más específicamente a continuación, tales medidas de centralidad y de agrupación se han seleccionado para analizar la posible coincidencia o divergencia de los agentes con posiciones centrales, tal como lo hemos definido teóricamente en el primer apartado de este artículo, de aquellos con un control o centralidad local.

\section{A) Centralidad de rango}

El rango da el nombre total de relaciones de un nodo con otro: una multiplicidad de fuentes informativas o de nodos de conexión puede suponer una mayor posesión de recursos de signo cognitivo y, por tanto, una mayor acumulación in situ de información. De esta forma se produce, si es el caso, una clara participación de la 
distribución de conocimiento compartido y mayores alternativas y oportunidades para incidir en la secuencia de intencionalidades.

El índice de poder de Bonacich, por otro lado, es una medida complementaria que incluye en la jerarquía el poder de las conexiones no directas, que nos puede ser muy útil para analizar las situaciones de distribución de conocimiento coordinadas y complejas, como las de un rodaje cinematográfico. En efecto, veremos cómo no existen unas claras jerarquías ni relaciones unidireccionales, dada la alta densidad comunicativa e interactiva de la red técnico-funcional.

\section{B) Centralidad de proximidad}

Como es sabido, con el cálculo de proximidad se obtiene así un grado de conexiones entre los agentes que nos da cuenta de la proximidad o no de la relación, primero, para intercambiar información en el caso de las dos primeras redes $\mathrm{y}$, segundo, para manipular instrumentos como en la tercera. Los nodos centrales, los más cercanos al conjunto de los otros nodos, pueden ser un punto de referencia para los otros agentes en el sentido de su accesibilidad y de dichos nodos con relación al resto en el sentido. Se trata de un indicador de alta capacidad de control local, aunque la toma de decisiones no tiene por qué ser central en el proceso de representación, como veremos en el último apartado del artículo.

\section{C) Centralidad de intermediación}

La capacidad de intermediación puede otorgar poder y prestigio, aunque también puede provocar sobrecarga informativa o ruido por diferencias en las fuentes. Los agentes intermediarios, por lo tanto, se encuentran en múltiples canales informativos, y con frecuencia se ven a sí mismos como aquellos que hacen que las cosas se produzcan, es decir, como negociadores de la situación a la que se enfrentan.

Como también veíamos en la medida de rango con el índice de poder de Bonacich, con el flujo de intermediación vemos el grado en que un agente se involucra en el flujo informativo entre las parejas de agentes de la red, y tenemos en cuenta los itinerarios que conectan a los nodos más allá de los caminos geodésicos.

\section{D) Medidas de agrupación}

Si las medidas de centralidad nos dan pistas acerca de la co-presencia y yuxtaposición de nodos en la red, en función de la cantidad, el tipo y el origen de las interacciones de los agentes, podemos observar también el grado de sociabilidad (solidaridad) y de adhesión (accesibilidad) del contenido informativo y cognitivo de la red. 
Así, con los cliques se definen los grupos en que se dan el máximo número de relaciones posibles entre sus miembros, conectados por todos lados. Los $\mathrm{n}$-clan indican los grupos de miembros con una relación de $\mathrm{N}$ pasos, que limito a 2, para incluir agentes con una relación indirecta. El k-plex dibuja los grupos en que los agentes establecen relaciones directas con $\mathrm{K}$ agentes externos al grupo, y que también limito a 2 . Se hablará en los dos casos de grupos con 3 miembros, para superar la díada y dar más juego combinatorio. Para terminar, estableceremos 6 facciones o particiones de los datos de las tres redes, más o menos en correspondencia con las áreas profesionales que se han indicado más arriba: dirección, producción, fotografía, atrezzo, sonido, y eléctricos. Aplicando este último análisis veremos si las relaciones comunicativas de la red siguen las particiones profesionales establecidas antes del comienzo de la actividad de rodaje.

Una vez descritas las técnicas utilizadas, daremos cuatro pinceladas acerca de la naturaleza de la red técnico-funcional de agentes intencionales, que como venimos diciendo, constituye una forma de abordar las relaciones de producción de conocimiento distribuido y de comprender así el trabajo real de producción cinematográfico.

\section{Un compendio del material analizado y sus apuntes teóricos}

Como decíamos en el primer apartado de marco teórico, el rodaje cinematográfico es un proceso de representación en el que agentes intencionales toman decisiones coordinando sus acciones, en una sincronización de tareas y de recursos representacionales que hacen emerger la complejidad del proceso. Veamos pues a partir de los resultados la naturaleza del proceso estudiado, definiendo cuatro atributos de la red técnico-funcional: sincronización, coordinación, simultaneidad y complejidad.

En primer lugar, tenemos que determinar si efectivamente existe, a partir de la plasmación por redes de los tres tipos de interacciones, comunicativas, informativas e instrumentales, una dimensión de sincronización en el trabajo observado. Es decir, si los agentes, de forma simultánea, a lo largo del rodaje de la escena cinematográfica, manipulan y se intercambian recursos diversos, de forma que la sincronización de sus interacciones sea necesaria para asegurar la unidad comunicativa y la estabilidad del producto, es decir, de la escena cinematográfica. También es un elemento susceptible de sincronización la presencia de elementos contextuales, que mencionábamos en el apartado conceptual, provenientes del campo cinematográfico y que tienen a veces que ser tomados en consideración en el desarrollo de las tareas de producción. 
En este sentido, las medidas de centralidad han confirmado la existencia de una dimensión de coordinación central en el desarrollo objetivo del proceso de representación. La ayudante de dirección, seguida a distancia por los miembros de producción ejecutiva, ejerce la mayor parte de las tareas de coordinación, que se refleja en la cantidad de interacciones verbales profesionales e informales que se intercambian en la segunda red, la centralidad de rango más alta; su posición como punto de mira de los intercambios verbales, la más alta centralidad de proximidad; y su posición privilegiada como negociadora de intercambios verbales profesionales e informales, como nos indica la más alta centralidad de intermediación.

La densidad moderada de las dos redes comunicativas, sobre todo de la informacional o profesional, con un $50 \%$ de densidad global, verifica la elevada simultaneidad de las interacciones, si entendemos por interacciones simultáneas las que se dan en el proceso observado, de 3 horas, de filmación de una escena cinematográfica. Esta tendencia se ve reforzada por la baja centralidad global de la intermediación, alrededor del $18 \%$ en relación a la red estrella, que muestra la poca necesidad de relaciones indirectas, a resultas de elevada interacción directa entre los miembros del equipo del rodaje, así como por la presencia de agrupaciones difusas, 3 o 4 según la red, de agentes conectados por un mínimo de 2 pasos que incluyen la práctica totalidad de la red, entre 25, en la red informacional, a 31 miembros del equipo en la red comunicacional. En estas agrupaciones vemos también cómo siguen siendo los miembros de los sub-equipos de dirección, producción ejecutiva y los actores, aquellos agentes que más se repiten. Otro indicador del elevado número de interacciones surge de la multitud de pequeñas agrupaciones de más de 3 miembros que se pueden formar en las dos redes verbales.

Por lo que a los recursos compartidos e intercambiados se refiere, más allá de los recursos de signo cognitivo verbal que hemos comentado hasta ahora, vemos con la tercera red, la instrumental, cómo aquellos nodos que están más distribuidos y, por lo tanto, tienen un mayor rango, proximidad e intermediación, son los recursos de valor utilitario, es decir, los más ligados a funciones que traspasan el proceso concreto de representación cinematográfico para formar parte de otros campos de la actividad social, como es la alimentación, a través del catering, y el hábito de fumar, con el intercambio y uso de cigarrillos. También poseen un grado de centralidad apreciable los recursos eléctricos y de decoración-atrezzo. Si los primeros, los eléctricos, tienen un alto valor utilitario, tenemos que destacar sobretodo el valor de signo cognitivo que comparten con los segundos, ya que están implicados en el proceso de representación de ficción de una forma directa. El 
material eléctrico asegura el funcionamiento de los instrumentos cognitivos centrales, con valor dominante en el entorno, como la cámara, el vídeo o el enfoque, que caracterizan el arte cinematográfico, como decíamos más arriba, al citar a Walter Benjamin. Por otro lado, los diversos elementos escénicos que constituyen el set en donde los actores realizan la acción de ficción, y que integran muebles, vestuario, carteles, etcétera, contribuyen a transformar el espacio físico del rodaje hacia un espacio de ficción, elemento también indispensable para llegar a la producción creíble de una escena cinematográfica.

A partir de las medidas de agrupación, hemos visto que las agrupaciones obtenidas son amplias y poco fragmentadas, por lo que podemos decir que el grado de sociabilidad (solidaridad) y de accesibilidad de la información distribuida es elevado. No obstante, recordemos que hablamos en todo momento de las informaciones intercambiadas verbalmente y no del conjunto de recursos de signocognitivo en posesión de los agentes del proceso. Como introduciremos en el apartado siguiente, sin entrar en ello ahora, los agentes con recursos de signo cognitivo más valorados en el campo cinematográfico artístico, como son el director y el cámara, no ocupan posiciones destacadas en la jerarquía de centralidades, así que parece que ciertos recursos no forman parte de esta red de distribución de conocimiento situado.

En cuarto lugar, hemos observado también características de complejidad en el rodaje. Como ya hemos visto, el número de interacciones entre los agentes es elevado. Además, los recursos en juego son de naturaleza diversa, como hemos visto a partir de la tercera red instrumental. La dependencia instrumental es generalizable a todos los miembros de la red, dado la centralidad de rango elevada de los instrumentos y demás recursos no-verbales. Si entramos en detalle, vemos que los grados de distribución es diferenciada, y que el tipo de recurso también varia mucho según la función del agente que lo manipula.

También debemos tener en cuenta que la red informacional no tiene las mismas medidas de centralidad ni de agrupación. Por ejemplo, si los actores ocupan posiciones centrales en la red informal o comunicacional, en la red profesional o informacional desaparecen, mientras que, a la inversa, el guionista, que es un elemento contextual que no pertenece al entorno, no aparece en la primera red, pero suma posiciones en la profesional, al interactuar con agentes centrales. Si observamos las facciones en las dos redes, vemos que aún estando las agrupaciones profesionales marcadas desde pre-producción, existen dos factores sociales que modifican su composición. 
Por un lado, en la primera red informal los agentes se agrupan según factores de edad o de posición jerárquica dentro del correspondiente sub-equipo. Así, los jefes de producción y de dirección se separan de sus ayudantes, que integran otra facción. También entra en juego la misma dinámica del rodaje, y así se pueden crear grupos de trabajo in situ según la necesidad que se tenga, en el momento, de coordinar diversas acciones. Por ejemplo, la ayudante de dirección, los actores y el jefe de producción forman una facción dentro de la primera red de comunicación informal.

De forma inversa, agentes que se podrían agrupar, para desarrollar tareas en un mismo espacio y tiempo, se mantienen separados, con poca interacción o sin compartir información, trabajando de forma paralela. Un ejemplo de tal proceder se da entre el equipo de sonido y el de fotografía: aún trabajando con recursos parecidos, y ser el primero un equipo de trabajo con solo dos miembros, se mantienen separados y sin compartir tareas ni información. La baja centralidad de los miembros del equipo de sonido, y su constitución como facción profesional separada de las otras, contrasta con su papel central en el proceso de rodaje, ya que son los únicos encargados de la producción de la banda de sonido. Su poca participación en la red técnico-funcional, su bajo nivel de interacción y de intercambio de recursos de signo cognitivo verbal, se explica entonces por la existencia de subprocesos paralelos dentro del mismo proceso.

Es decir, los agentes de sonido pueden desarrollar sus funciones profesionales sin tener demasiado contacto verbal con el resto de miembros de la red. Este caso se repite con la técnica de vídeo, dentro del equipo de fotografía: si en la red comunicacional básica ocupa una posición de centralidad cercana a la mayoritaria, en la red informacional vemos como cae en una posición periférica. Probablemente, la función de control del vídeo y de sus monitores corresponde a un tercer subproceso, que se desarrolla en solitario, con los instrumentos cognitivos correspondientes.

Existe un tercer grupo de agentes que tampoco participan en los niveles medios de centralidad, y que parecen manifestar también esta no-correspondencia entre la atribución formal de funciones y la interacción real entre los agentes. Los bajos niveles de interacción del director y del cámara, no se pueden explicar por un proceso de trabajo en paralelo, sino que es consecuencia, como veremos en el apartado siguiente, de la naturaleza de los recursos representacionales con los que trabajan. 
Finalmente, un cuarto elemento completa la dimensión de complejidad que estamos comentando: el grado de libertad de los agentes en la realización de las tareas asignadas. Así, agentes periféricos, como ciertos agentes de electricidad, de sonido y de maquillaje, parecen estar alejados del proceso, con bajos niveles de intermediación y de rango. Por un lado, estos resultados nos indican una cierta intercambiabilidad de los agentes que también se refuerza por su presencia en el entorno, en cierta medida, intermitente. Solo los agentes centrales tienen que estar de forma permanente en el rodaje, por ser los ejes de la coordinación del proceso, mientras que aquellos agentes menos centrales pueden entrar y salir del entorno con más libertad, e intercambiarse con otros miembros del mismo equipo. Probablemente este hecho proviene de la diversidad de recursos manipulados en el proceso, aunque vemos que estos agentes son pocos y siguen la tendencia comunicativa de los otros agentes, es decir, que se mantienen en niveles de interacción elevados.

Resumiendo, podemos decir que la red técnico funcional es sincronizada, coordinada y compleja a partir de los elementos analíticos enumerados hasta ahora. Tales características son el resultado cognitivo y, por lo tanto, social de las interacciones locales de agentes intencionales, tal como hemos expuesto en el primer apartado del artículo.

\section{I. Los límites del proceso y el carácter central del director}

A continuación, y antes de pasar a las conclusiones de este artículo, hablaremos brevemente de una característica de la red técnico-funcional observada, y que se encuentra en relación con la distinción teóricamente elaborada entre agentes centrales y periféricos, según los recursos representacionales manipulados sean dominados o dominantes en el entorno.

\section{Centralidades diferenciadas entre agentes: el director y los otros en sus variedades}

En efecto, vemos cómo los agentes con los recursos de signo cognitivo artístico, centrales en el proceso de representación, como el director y el cámara, muestran bajos niveles de interacción y, por lo tanto, de centralidad, así como poca presencia en las medidas de agrupación.

Concretamente, el director ocupa en todas las redes posiciones totalmente periféricas, muestra de su bajo nivel de interacción, de comunicación y de participación en la coordinación de tareas en el rodaje. Solamente en el flujo de intermediación lo encontramos más cercano a la media de intermediación, que seguramente se deriva del tándem interactivo existente entre la ayudante de dirección y el director. Es dicha ayudante la que ejerce las máximas funciones de 
coordinación y de comunicación en la red, y la que ocupa la máxima centralidad en todas las redes comunicativas: de alguna forma, parece que se podría hablar de delegación de funciones, en el sentido que, mientras la ayudante domina el nivel de relaciones comunicativas, coordinando recursos y agentes, el director es quien toma las riendas de las secuencias intencionales de decisión.

En definitiva, como veremos a continuación al hablar de los límites del proceso, el director, independientemente de su débil control local, es quien toma las decisiones dominantes en la organización del rodaje en tanto que producción artística. Para completar la explicación, haría falta echar mano de la naturaleza de la doxa del campo cinematográfico: de momento sí que podemos afirmar que el director, junto con el cámara, es quién posee los recursos de signo-cognitivo artísticos más valorados en el campo, y por tanto, dominantes en el entorno. En la observación del proceso hemos encontrado numerosos ejemplos de la exclusividad de las tomas de decisión acerca de la acción de ficción, sobre la localización y la acción de los actores, de la ambientación, del movimiento de la cámara, etcétera. Así, al limitar su alcance, los recursos artísticos no se hacen comunicables con el resto de la red de agentes, excepto con los actores- que prácticamente son recursos manipulados por el director- y el cámara, imprescindibles para la tarea de la filmación de la ficción.

En las medidas de agrupación, vemos cómo el director no sigue el mismo patrón que los otros jefes de las sub-áreas de trabajo no formando parte de las pequeñas agrupaciones, si bien participa de las más difusas, gracias a la intermediación de la ayudante de dirección. Dentro de la división del trabajo, lo encontramos junto al guionista y la script: el primero, como hemos dicho anteriormente, es un agente contextual que no cumple una función estable en la red técnico-funcional pero que, sin embargo, posee un recurso también dominante en el campo cinematográfico como es el guión. Éste constituye una guía para las tomas de decisiones artísticas del director, ayudado también por la script, que prácticamente es un complemento del guión. Estos tres agentes, por lo tanto, resultan centrales para el rodaje, aún siendo invisibles en las redes comunicativas, debido a la apropiación y dominio que ejercen sobre los recursos de naturaleza artística a los que están vinculados.

Por lo tanto, las representaciones de las redes que hemos presentado precedentemente, que muestran el rodaje como una red distributiva de recursos e información, esconden realmente la dimensión artística del proceso. Aunque esta dimensión está plenamente condicionada por el campo, su naturaleza es restrictiva y poco accesible a la mayoría de los miembros del equipo del rodaje. En consecuencia, existe una clara jerarquización según el grado de apropiación de 
recursos artísticos, apropiación que se superpone a las observaciones reticulares realizadas que muestran una aparente uniformidad y distribución de intercambios comunicativos en el rodaje. Dicho poder se manifiesta en el carácter central del director y no es visible directamente en las redes.

\section{La clausura del proceso y los poderes del director}

El análisis de redes, añadido al material procesado en forma de notas de campo, ha permitido definir el rodaje de una escena cinematográfica como un proceso de representación acabado. En efecto, el conocimiento socialmente distribuido necesario para la red técnico-funcional del rodaje se basa en una distribución de recursos- parcial, como hemos visto-, en una mutua comprensión entre los agentes y en un clima amable e informal que facilita la unidad comunicativa. Además, la escena resultante mantiene un cierto grado de estabilidad por encima de las diferencias técnicas en el soporte de la filmación, que puede ser digital o analógico, y de las diferencias en longitud, según el número de tomas filmadas. El rodaje tiene lugar en un espacio y un tiempo fijado por la localización y el horario negociado desde producción y realización. $Y$ además, existen unos cargos o posiciones de los agentes participantes en la red de producción que se mantienen invariables, por encima de la intercambiabilidad de los agentes periféricos.

Más allá de estos cuatro factores que definen el proceso de representación (unidad comunicativa, estabilidad del producto resultante, espacio-tiempo común, y presencia invariable de los agentes), existe un quinto factor que da visibilidad a la desigualdad social de toda interacción cognitiva. En efecto, en toda situación social la diferenciación entre agentes participantes y los iniciadores / 'finalizadores' del proceso, resulta pertinente e ilustrativa: por un lado, porque dicha diferenciación, más concretamente los 'finalizadores', marca los límites de la unidad de análisis establecida para nuestro objeto de estudio, y por otro lado, porque es uno de los indicadores más característicos de la centralidad del director.

Como introducíamos anteriormente, la decisión más crucial en el proceso, que es la de definir el proceso de rodaje, proviene virtualmente de un solo agente del entorno, el director de la película. Las fases de preparación del rodaje están fijadas por la hoja de trabajo y se elabora desde producción, por otro agente central contextual, que posee los recursos económicos dominantes en el campo. No obstante, el rodaje sólo se inicia al reunirse los agentes en el lugar prefijado del rodaje. Cada toma requiere un proceso de preparación de los recursos y de los agentes: estas fases de preparación exigen la participación de todos los miembros de la red técnico-funcional. 
El comienzo del rodaje lo marca directamente la ayudante de dirección verbalmente, con una frase que a pesar de ser un lugar común, es funcional dado que coordina el inicio de los distintos subprocesos del rodaje (Silencio, sonido, cámara y acción). Así, se pide confirmación verbal por parte de los jefes de sonido, vídeo y fotografía, por lo que dicha frase 'consagrada' en el argot cinematográfico, manifiesta y es el resultado del carácter distribuido de las condiciones materiales, funcionales, cognitivas y sociales del rodaje. En efecto, los distintos subprocesos técnicos tienen que estar a punto para que el rodaje de ficción pueda comenzar. También tienen que estar preparados los actores y el director, que es quien comunica a la ayudante de dirección la conveniencia de empezar a rodar. A través del walkie o a viva voz la ayudante pide confirmaciones, el runner se pone delante de la cámara con la claqueta y recita la información que hay escrita (número de escena, de toma, número de cinta utilizada para la grabación, posición de foco de la cámara, y frecuencia de la banda de sonido), información que se repetirá al inicio de cada toma. Por lo que al final de cada toma se refiere, éste se produce siempre cuando el director verbalmente dice "corten" y habla con su ayudante, que es quien transmite en voz alta, o través del walkie, la orden de finalización al resto de agentes participantes.

Si el inicio del proceso está tan condicionado por la voluntad artística del director y de los actores como por las condiciones de las posibilidades materiales que aseguran los miembros de la red técnico-funcional del rodaje, el final de la toma del rodaje proviene exclusivamente de la decisión personal de director. La decisión del director acerca del número de toma rodada para una escena así como su prolongación y duración como proceso, se justifica a partir de sus recursos poseídos de signo cognitivo-artístico. Una vez el director decide la inclusión o no de una toma en la escena rodada y su validez o calidad estética como parte del producto final, entrarán en acción el resto de agentes del proceso para modificar elementos técnicos del proceso, de cara a la toma siguiente.

El director es el único con una doble intencionalidad, que incluye, además de la acción intencional compartida en el proceso con los otros agentes participantes, la de acabar un objeto de arte. En el caso que nos ocupa, consiste en guardar como atribución suya la relación con dicho contexto y con el campo, delimitar la unidad y entidad del rodaje y elegir e identificar, esto es definir, la escena final además de apropiarse, dentro del campo, de la escena resultante. Es precisamente esta segunda intencionalidad lo que diferencia el director del resto de los agentes, y como comentamos en el primer apartado, esta diferencia no proviene del desarrollo funcional del proceso (otro agente podría hacerlo), sino de la posición profesional 
que el director ocupa según la estructura del campo cinematográfico al que pertenece.

Si el director es el máximo representante del carácter exclusivo y dominante de los recursos de signo cognitivo artístico y de la personalización de la toma de decisiones en el proceso de representación, el caso del director de fotografía, que en este caso es la misma persona que el cámara, presenta diferencias interesantes. También se trata, en este caso, de una figura invisible en el análisis de redes, aunque las decisiones intencionales del cámara no son solitarias. Es decir, al manejar recursos artístico-técnicos como la cámara, el enfoque, los filtros, etcétera, depende de los instrumentos cognitivos y de los recursos de los agentes de eléctricos, como cables, equipo de iluminación, etcétera, así como de sus ayudantes del equipo de fotografía que dirige.

Por lo tanto, en la toma de decisiones, el cámara muestra cierta interacción comunicativa con sus ayudantes y con los otros miembros del rodaje. Este mayor intercambio informativo y esta mayor dependencia instrumental se refleja en el análisis de centralidad de intermediación, en el que se sitúa un poco por encima de la media, y en la copresencia, en las facciones, con otros miembros de su equipo de fotografía y con los jefes de producción y otros miembros de dirección. No obstante, el alto valor en el campo de sus recursos lo sigue aislando de la red de conocimiento distribuido, de una forma parecida al director.

\section{Conclusiones}

Hasta aquí hemos introducido teóricamente nuestra postura acerca del rodaje cinematográfico como un proceso artístico de producción y nos hemos planteado la pregunta acerca de la naturaleza real del rodaje, es decir, acerca de las secuencias de intencionalidades de los agentes que hacen posible un conocimiento socialmente distribuido y una coordinación de interacciones para llegar a la producción de un resultado, que en el caso observado es una escena cinematográfica.

Tal como veíamos en la síntesis teórica del apartado de análisis de redes, el conocimiento producido en el rodaje es interactivo en la medida que es intencional primero, y sincronizado, coordinado, simultáneo y complejo, segundo. Así, las tres redes trabajadas nos han dado una representación gráfica de los agentes participantes con un mayor control local y un dominio del conocimiento técnico y social necesario para coordinar las tareas complejas de un rodaje. Este primer nivel de análisis es el primer eslabón para hablar del conocimiento y de la acción social desde un punto de vista intersubjetivo y distribuido, ya que observamos cómo la 
producción de una escena cinematográfica es un producto de equipo, y no un resultado de una actividad mental aislada.

No obstante, vemos que existe una confrontación, o un contraste, entre los agentes centrales de la red funcional y los agentes que definen el producto final, la escena cinematográfica, y que toman las decisiones centrales de ficción, que son principalmente el director y el cámara. En efecto, al tener en cuenta, más allá del entorno inmediato de la actividad del rodaje, el marco social general, correspondiente al campo cinematográfico, vemos cómo las decisiones de los agentes vienen condicionadas no solo por su tarea en la red técnico-funcional, sino sobre todo por el tipo de recursos representacionales que poseen y que se valoran de forma diferenciada en el campo cinematográfico.

Como decíamos al principio de este artículo, un análisis sociológico, ante todo, busca los mecanismos de poder de cualquier proceso productivo. Para mejor responder a la pregunta de qué beneficia a quién, hemos elaborado dos conceptos o mecanismos que surgen o se infieren claramente del análisis de la observación del rodaje a partir de las redes sociocéntricas; lo denominaremos como "la paradoja del director y la dualidad cinematográfica".

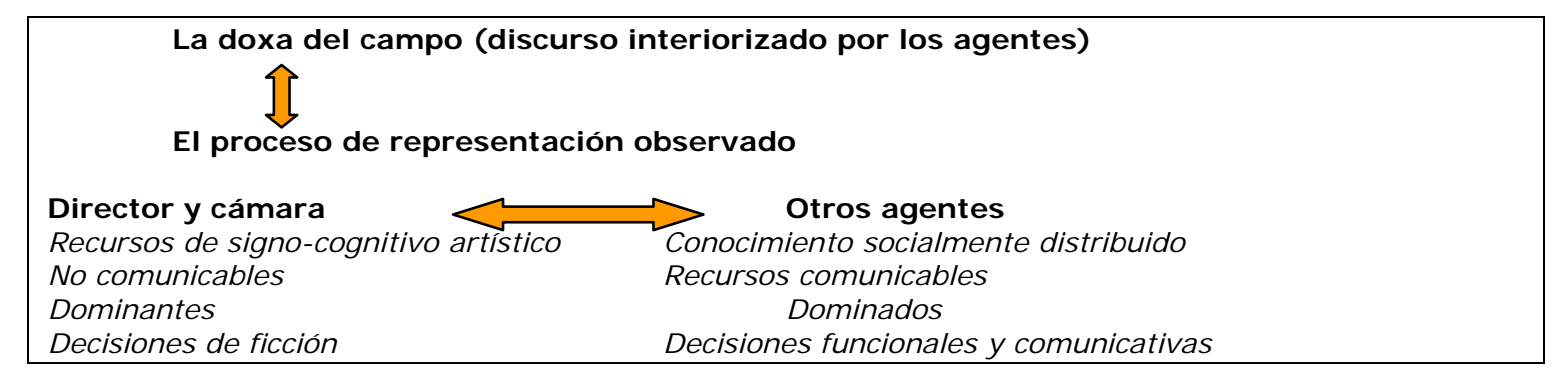

I lustración 6. La paradoja del director

El primer mecanismo surge de la contradicción existente entre el tipo de recursos representacionales poseídos por el director y el cámara, agentes centrales, y los recursos de los otros agentes participantes. Como vemos en la Tabla 1, y como se plasma en el análisis de redes sociales, la capacidad de decisión sobre la identificación del rodaje como proceso artístico, que equivale a la posesión de la doble intencionalidad acerca de la creación del resultado final, la escena, recae exclusivamente en el director y en el director de fotografía o cámara. Por lo tanto, el poder de dominación de los agentes centrales surge de la configuración del campo y no de la idiosincrasia de los agentes o de la organización técnico-funcional de la red cognitiva y comunicativa del proceso.

\section{Traducción de la negación del principio de homología}

El director y el cámara

Condiciones ficcionales de representación 
El mecanismo social de la dualidad cinematográfica plasma el porqué de la paradoja del director: el campo cinematográfico se define por dos tipos de capital, el económico, en manos de la producción ejecutiva, y el artístico, específicamente en manos de realización. Si bien el campo cinematográfico es una industria, se rige también como un campo de producción artística. Y, como vemos en la observación del entorno del rodaje, es central un principio de negación de homología que pasa por alto las condiciones técnico-funcionales de la producción cinematográfica, para valorar y poner de relieve el proceso doblemente intencional del director y del cámara. Es decir, socialmente se define el rodaje como proceso creativo de unos agentes, sin tener en cuenta su dependencia de una red técnico-funcional de los agentes que participan del proceso real del rodaje.

Tales mecanismos sociales, por lo tanto, aíslan al creador, al artista, de sus circunstancias sociales, de las condiciones reales de representación, que es lo que hemos venido observando. Así, el análisis reticular ha definido los agentes con un control local de la red técnico-funcional del proceso, ha afirmado empíricamente la naturaleza colectiva de la realización de una película, y ha definido las dimensiones de coordinación, de sincronización y de complejidad del rodaje.

Sin embargo, y para corroborar aún más estas conclusiones que tienen que ver con la relación del entorno inmediato del rodaje con su articulación en su campo y contextos, insistimos en el hecho de que un análisis sociológico de la producción cognitiva en un entorno productivo, sea un rodaje cinematográfico, sea cualquier otro entorno productivo, requiere ir más allá del análisis local de redes y completar las observaciones con aportaciones teóricas y empíricas acerca de la existencia de campos sociales, de mercados en los que los agentes se encuentran en situaciones de desigualdad.

En definitiva, el análisis de los recursos intercambiados y poseídos, más allá de las relaciones de interacción y de comunicación cognitiva, es necesario para entender las cadenas de intencionalidades, la toma de decisiones de los agentes en todo proceso de producción.

\section{Bibliografía}

Barab, Hay, Yagamata- Lynch (2001). “Constructing Networks of Action- Relevant Episodes: An In Situ Research Methodology", The Journal of the Learning Sciences; 10, 63- 112. 
Bardram, J. (2000). "Temporal Coordination" Computer Suported Cooperative Work, 9, 157-187.

Benjamin, W. (1983). L'obra d'art en l'època de la seva reproductibilitat tècnica, Barcelona: Edicions 62.

(1998) Les règles de l'art; Paris : Seuil.

- (1994) Raisons Practiques; Paris : Seuil.

- (1979) La distinction : Paris : Les Éditions de Minuit.

Bourdieu, P. y Chamboredon, Passeron (2000). El oficio del sociólogo. Mexico DF: Siglo XXI.

Bourdieu, P. y Wacquant (1994). Per una sociologia reflexiva. Barcelona: Herder.

Cicourel, A. (2002). « La gestion des rendez-vous », ACTES de Récherche, 143.

Dreyfus, H. L. (1993). What Computers Still Can't Do. Cambridge: MIT Press.

Engeström, Y. y Midlleton, D. (eds.) (1996). Cognition and Communication at Work. Cambridge UP.

Flyuberg, B. (2001). Making Social Science Matter. Cambridge UP.

Geertz, C. (1974). Local Knowledge. New York: Basic Books.

Giere, R. y Moffat, B. (2003). "Where the Cognitive and the Social Merge", Social Studies of Science, 33/2, 1-10.

Hutchins, E. (1995). Cognition in the Wild. Cambridge: MIT Press.

Jonasseen, D.S. y Reeves, A. (eds) (1993). Handbook of Research for Educational Communication and Technology; New York: Simon i Schuiter Mc Millan.

Lozares, C. (2005). "Proceso de trabajo y contextos" Revista PAPERS, Universitat Autònoma de Barcelona, en publicación.

Luz González,A; y Molina, J.L. (2003). “Introducción: Redes para pensar lo social” Revista REDES; 4-junio, ISSN 157 9-0189 www.redes-sociales.net

Pettit, P. (1986) The Common Mind; Oxford UP.

Searle, J. (2004) Mind; Oxford UP.

- (1983) Intentionality; Cambridge UP.

Sperber, D. y Wilson, D. (1995). Relevance: Communication and Cognition; Oxford: Blackwell Publications.

Valsiner, I. y Van der Veer, J. (2002). The Social Mind; Cambridge UP. Vygotsky, L. V. (1962). Thought and Language ; Cambridge: MIT Press.

Zhang, J. y Norman, D.A. (1994). "Representation in Distributed Cognition Tasks", Cognitive Science, 18, 87-122. 